background radiation are interesting, but I think that they are given misleading and misplaced prominence in a book of this nature; after writing with unusual care and clarity for three-quarters of the way, it seems to me that Layzer has over-indulged himself. As a volume in the Scientific American Library series, a large number of people will read this book. It is a pity that having done so, they will know nothing of the exciting observations and theories that underpin our most recent ideas about the construction of the Universe. This is a book that could have been outstanding - what a sadly wasted educational opportunity.

It is difficult to know what to say about John Boslough's "'biography" of Stephen Hawking, written, I gather, without its distinguished subject's blessing. Certainly, it is written in a style reminiscent of certain dubious tabloid newspapers. The book is built around a collection of quotes and anecdotes gleaned at first- and secondhand from sporadic conversations and interviews, but like many pieces of semisensational journalism the author is interested only in the extraordinary and the remarkable. This approach may work well when reporting the Cup Final but in any work of biography it is disastrous. Here, the real picture of Hawking's character and ideas is superseded by a patch-work of aphorisms and anecdotes that are atypical, for indeed that is why they are so memorable. Why has the author not interviewed Hawking's many collaborators and associates with the goal of presenting an accessible but thorough and accurate description of his way of thinking? Accessible need not mean trivial.

The author's handling of the scientific ideas involved is unreliable and I am sure, to anyone not completely familiar with what he is trying to explain, unintelligible. Buzz-words and acronyms spontaneously appear without explanation - "GUT's", "flatness", “inflation", "Guth's scenario" - while the melodramatic style leads to errors and absurdities: Einstein's special relativity paper was not a "bombshell"; he did not show that "time did not always flow from past to present"; the concept of a black hole horizon did not arise as a consequence of Hawking's Area theorem, nor is its surface area the only externally observable property of a black hole. Then there is the description of how Einstein discovered the mass-energy formula: "Einstein came to the conclusion that $m=E / c^{2}$. From there it was just a simple algebraic step to the most famous equation in history, $E=m c^{2}$ ".

Perhaps some will enjoy this book. I didn't. Stephen Hawking is one of the world's most remarkable scientists and I hope that a biography worthy of his achievements will one day be written. This, I am afraid, is not it.

John D. Barrow is a Lecturer in Astronomy at the University of Sussex.

\section{The best lab in the world?}

\section{Nevill Mott}

Three Degrees Above Zero: Bell Labs in

the Information Age.

By Jeremy Bernstein.

Charles Scribner's Sons: 1984. Pp.241. $\$ 17.95$.

BELL Laboratories are envied worldwide, and Bell scientists know it. Of what other industrial laboratory could the vicepresident for research write:

if I hire the fifth-best theoretical physicist in the country I can get away with it, but if I hire the fifteenth-best I am wasting my time. We are looking for number two or three, and we want to compete with the best universities for number one.

One can sometimes notice at conferences that the Bell scientists sit and talk together, and those from other laboratories sit elsewhere. What other industrial laboratory has had seven Nobel Laureates since the War? And certainly no other laboratory has been funded by the company (AT\&T) with a monopoly of telecommunications in the United States.

Jeremy Bernstein's book gives a picture of Bell Labs by describing certain projects and some men and one woman at the centre of its achievements. It is written, too, at a moment when the future is in doubt, and uneasiness about the coming years colours many of the articles in it. Just two years ago an agreement was signed between the Justice Department and AT\&T which split up the telephone company but allowed it to retain Bell Labs. Fears are expressed that fundamental research may be squeezed, results expected too quickly, supervision become too close and that Bell Labs will become "like any other industrial laboratory"'. Some of the scientists who feature in the book are optimistic but the anxiety comes through only too clearly. Those who have watched events from across the Atlantic have wondered whether it was worth while, so as to introduce more competition, to put at risk the quality of, perhaps, the best laboratory in the world.

I do not know whether Bernstein wrote this book because of what had happened to the telephone company; he does not say so, and it is not his main theme, though perhaps the most pertinent one. His aim is to tell a story. He writes of the early days of the laboratory, and then, inevitably, about the transistor.

This story has been told elsewhere, for instance in Braun and MacDonald's Revolution in Miniature, published by Cambridge University Press in 1978, and the author acknowledges what a fine study that book is. Reading Bernstein's own account, it seems certain that no organization but Bell could have made the breakthrough, at any rate not until years later. Even before the War, the need to replace the vacuum tube was recognized. The achievement, through the understanding of theory coupled with the most practical advances in materials science, could have occurred only in a place where the need was urgent and where all these skills existed. Even so, it was slow to come to fruition. The discovery was in 1947; it was the end of the 1950s before the electronic revolution was really underway.

Perhaps the most interesting pieces in the book are the vignettes of some of the people active now. The book had its origin in a discussion between the author and Philip W. Anderson, who shared the Nobel prize in 1977 with his former teacher J.H. Van Vleck and the present reviewer. An outstanding paper of Anderson's, which started the applications of solid state theory to electronics in glasses, was called "Absence of Diffusion in Certain Random Lattices", published in the Physical Review in 1958, and is referred to in the book's "Selected Bibliography". As the book is meant for non-specialists and indeed for readers who are not scientists at all, I should warn them that even for experts this is an exceptionally difficult paper; Anderson himself said it was "often quoted but never read". Its results were frequently disbelieved, but once accepted it was extraordinarily illuminating and, with a time delay of nearly ten years, sparked off a burst of theoretical work on noncrystalline semiconductors. Of course this work continues with great vigour at present.

Of interest too is the sketch of Anderson's career. It is surprising how hard it was for a theorist of genius, graduating from Harvard, to find a job in the immediate post-War years. But it was to Bell Labs he wanted to go, and he succeeded. Now, at sixty, he is something of an éminence grise, with a chair at Princeton and a role as adviser to the directorate of Bell. He has immense influence in American physics.

The woman in the story is Suzanne Nagel, described in 1970 when she joined the laboratory as "a very attractive, 27-year old, auburn haired Ph.D". Now she is one of many concerned with the glass fibre business. Of her current work, she told the author:

I have been especially concerned with wringing the last drops of water from the fibers. Very small amounts of water - parts per billion can cause attenuation of light in the spectral region where the lasers are emitting... Recently, by the way, we transmitted light for a hundred kilometers without the need for reamplification... The first transatlantic cable is due in 1988.

The title of the book refers to the temperature of the radiation background in outer space - the remains, so it is believed, of the energy dissipated in the Big Bang with which the Universe began $12 \times 10^{9}$ to $15 \times 10^{9}$ years ago. In fact the discovery of 
this radiation is perhaps the strongest evidence that the Universe did begin in this way, rather than as in Fred Hoyle's theory of continuous creation. The reader who doesn't know Bell Labs may well wonder what this has to do with an industrial laboratory, but after the War it was natural for a communications laboratory to hire a radioastronomer, and the consequence was the discovery of the background radiation by Arno Penzias and Robert Wilson. The management allows that sort of thing, and was rewarded when these two won the Nobel prize, shared with Peter Kapitza in 1978. As I remember from 1977, a Bell Nobel prize can be used as a song of triumph.

The last chapter is about Penzias himself. His father, born and resident in Germany, was a Polish Jew. In 1938 the Germans started deporting to Poland those who did not have German passports. Penzias and his parents got to the Polish frontier, but the Poles would not take any more people. After a brief period of imprisonment the family was sent back to Munich and told to get out in six months or else go to Dachau. Fortunately they succeeded in emigrating, and came to America. Education in New York's elementary schools for a boy who started with no English was tough, but Brooklyn Technical High School, New York City College and Columbia University under C.H. Townes led to Bell Labs, radioastronomy and the Nobel prize. Penzias is now vice-president in charge of research, an advance which he regards as "nothing short of miraculous".

This chapter on Penzias tells most about how Bell Labs is run and about fears for the future. On management he says:

We have to understand, and I think our owners do, that it can take years and years before someone may be able to make something useful out of a piece of pure research. That we understand this is one of the unique strengths of this place. The few competitors we have, who hire people as good as we do, do not do as well as we do because they manage them too closely.

The italics are mine, and it expresses the secret of Bell Labs' success. May it continue! Penzias's last words are: "I am a high pressure guy, and I didn't take this job to conduct a going-out-of-business sale".

Sir Nevill Mott is Emeritus Professor of Physics in the University of Cambridge. In 1977 he shared the Nobel Prize for Physics with J.H. Van Vleck and Philip W. Anderson, whose work is described in this book.

\section{Optics applied}

Springer-Verlag have issued a second edition of Matt Young's Optics and Lasers: Including Fibers and Integrated Optics, a revision of the original work of 1977.

The book is an introduction to the engineering and applied aspects of optics, and has been updated to take account of advances in optical communications and the development of integrated optics. Price in paperback is DM 64, \$22.

\section{Life, the Universe ... everything}

\section{C.J.S. Clarke}

The 4th Dimension: Toward A Geometry of Higher Reality.

By Rudy Rucker.

Houghton Mifflin: 1984. Pp.228. \$17.95. To be published in Britain in April 1985 by Hutchinson.

CAN the picture of the world developed by modern physics really affect the way people act and think? Clearly Rudy Rucker believes that it can, and to this end he sets out to teach the reader to think fourdimensionally. He achieves his aims mainly through a careful dimensional expansion of E. A. Abbott's romance about the twodimensional inhabitants of Flatland, published one hundred years ago, which he vivaciously continues in sections on "The Further Adventures of A Square'". Initially Rucker develops the idea of our space (in the common, three-dimensional sense) being embedded in a four-dimensional hyperspace, which allows for the possibility of space being curved, the whole exercise being carried out in a light-hearted style with copious cartoons. When he passes to space-time he can then build on the reader's understanding of four-dimensional geometry to describe the relativistic picture of the Universe as a four-dimensional manifold. In addition he can combine the pictures of space-plus-time and of space-in-hyperspace to move on to the idea of space-time as a whole being embedded in a five-dimensional hyperspace. Finally, with the inclusion of ideas derived from quantum mechanics, he presents a picture of the world as infinitedimensional, composed of the coordination of every inhabitant's separate perceptions of reality.

One great achievement of the book is that it should help to overcome the feeling of bemused awe by which non-mathematicians are often overwhelmed when multi-dimensional space is mentioned. On the one hand, the author succeeds in explaining clearly the way in which it can be useful to expand the mathematical description of space in terms of three parameters when further degrees of freedom are involved; on the other, he shows by discussing hyper-cubes and hyperspheres how the additional parameters can be built into a complete geometry.

But to convert the reader's heart and mind an author must do more: he must show that the higher spaces being built up are genuine geometrical wholes and not just arbitrary mathematical toys. In the case of special relativity Rucker conveys this well, showing that in the space-time of fast-moving particles the decomposition into space and time depends on what particle is used as the basis for a reference frame and so is not intrinsic to the spacetime itself: it is a true unity in which space and time have no absolute individual existence.

Though he is convincing on relativity, what are we to make of Rucker's higher space where the parameters being combined are as different in kind as (to take one of his examples) the raw-cooked and the sweet-salty axes for food? Surely here, whenever it is possible to define points in the space, it is also possible to decompose the space uniquely into the direct sum of the two constituent spaces. Before one can truly speak of a many-dimensional geometry, as opposed to the mere juxtaposition of lower-dimensional geometries, there must be some internal isotropy or symmetry between the dimensions.

This need for a symmetry between the dimensions becomes a crucial problem when it comes to the idea that our spacetime is embedded in a hyperspace. One of the main growth areas in theoretical physics is the study of Kaluza-Klein theories where just this happens. But the symmetry relating spatial directions with directions into the hyperspace is a supersymmetry that does not preserve the normal fabric of matter, and the size of the Universe in the other dimensions is generally taken to be minute. When Rucker talks of the world floating around in a hyperspace, attractive though the idea is, one must doubt whether there is any real connection between his speculations and those of the theoreticians. The weakness of Rucker's argument, at a macroscopic level, is that there is no parameter that could be used to define the additional dimension.

Another difference between the author's approach and that of modern theory concerns curved space. Rucker stresses the picture of the curvature of space-time being thought of as the curvature of a hypersurface in a hyperspace. By implication that hyperspace is flat (otherwise one would pass to a hyper-hyperspace to explain its curvature), whereas the hyperspace of modern theories remains curved. Sadly, no formal advantage has been derived from embedding space-time in some hyperspace, and it is not even known what is the minimum number of dimensions that such a hyperspace would have to possess in order to accommodate a general space-time: the best so far achieved is to show that it is less than 84 dimensions (see, for example, my paper in Proc. $R$. Soc. Lond. A 314, 417-428; 1970).

But $I$ am in danger of imitating the reviewer in the Times Literary Supplement who criticized Wind in the Willows as making a "negligible contribution" to natural history. Rucker aims not to write a monograph, nor indeed to present merely an amusing curiosity, but to make his ideas fire the reader's imagination and so make a difference to the way he thinks. To return to the question I posed at the outset, I believe that there is an interaction between 